\title{
Diagnostic accuracy studies needed for cone beam computed tomography
}

\author{
Abstracted from \\ Guerrero ME, Shahbazian M, Elsiena Bekkering G, Nackaerts O, Jacobs R, Horner K. \\ The diagnostic efficacy of cone beam CT for impacted teeth and associated features: \\ a systematic review. J Oral Rehabil 2011; 38: 208-216 \\ Address for correspondence: Maria E. Guerrero, Oral Imaging Center, School of Dentistry, \\ Oral Pathology and Maxillo-Facial Surgery, Faculty of Medicine, KU Leuven, Belgium. \\ E-mail: mariaeugenia.guerreroacevedo@med.kuleuven.be
}

\section{Questions: What is the efficacy of cone beam computed tomography $(\mathrm{CBCT})$ in the diagnosis of impacted teeth and associated features?}

Data sources Medline, Embase, CENTRAL and the reference lists of identified studies.

Study selection Studies had to describe diagnostic accuracy efficacy, diagnostic thinking efficacy, therapeutic efficacy or any combination for $\mathrm{CBCT}$ in the diagnosis of impacted teeth or of important features associated with impactions. Criteria for the diagnosis had to be described in detail or referenced. For studies elucidating only observer performance, the analysis had to be based on a minimum of two observers.

Data extraction and synthesis Data were extracted with the aid of protocol based on critical appraisal of diagnostic studies. Two authors independently assessed the quality and internal validity of studies using the QUADAS tool, ${ }^{1}$ with disagreements being resolved by discussion. The results were described narratively as meta-analyses could not be conducted. Results The search yielded 96 titles, of which seven were included. There was only limited evidence for diagnostic efficacy expressed as sensitivity, specificity and predictive values. Only two studies compared CBCT and panoramic radiographs with a valid reference method and presented the results in terms of percentage of correct diagnoses.

Conclusions There is a need for diagnostic accuracy studies on CBCT where accepted methodological criteria for diagnostic thinking, efficacy and therapeutic efficacy are incorporated.

\section{Commentary}

This systematic review is timely as there are approximately 5,000 СВСТ machines in the USA and the number is increasing. Concern about the orthodontic use of CBCT has been expressed: 'while there may be clinical situations where a CBCT radiograph may be of value, the use of such technology is not routinely required for orthodontic radiography (American Association of Orthodontists Resolution $26-10 \mathrm{H}, 2010)^{\prime}{ }^{2}$ In other specialties, such as endodontics, guidelines are emerging, ${ }^{3}$ but the critical question is if we have sufficient research of high enough quality to create guidelines. Guerro et al.'s review provides important information on the availability of high quality evidence regarding CBCT and impacted teeth.

The review is well written with a good overview of the prevalence of tooth impactions in different populations. The Material and Methods section is an excellent introduction to performing a systematic review, with sufficient detail and examples that general dentists in study clubs could replicate the technique with occasional guidance from an external consultant. The results indicate that a significant problem exists in the lack of good quality reports to create valid guidelines for the use of CBCT and impactions. However, this information is important as it will alert readers to be cautious when evaluating claims for the benefits of using CBCT. Although the review is limited to impactions it probably indicates the situation for other specialties. The new high resolution CBCT images with voxel sizes of $0.08 \mathrm{~mm}$ to $0.15 \mathrm{~mm}$ are providing good detail of periodontal ligament spaces. As a practising maxillofacial radiologist the problem is interpreting this new information since there are no answers to questions such as 'How do normal periodontal ligament spaces vary between the cervical and apical regions and from tooth to tooth?'

The review may also encourage dentists to question what are the risks and benefits of using СBCT compared to conventional radiography. In order to do this clinicians should know that the majority of practitioners in the USA still use round collimators and D speed film which produces a dose equivalent of 47 days of background radiation for 20 intraoral exposures. ${ }^{4}$ Some of the newer СВСТ machines produce doses between 3 to 10 days, considerably lower than a full mouth series. This may lead dentists to think that perhaps we can substitute CBCTs for intraoral radiographs. However, initial work seems to suggest that at this time CBCT is not a replacement for bitewing detection of caries. ${ }^{5}$

\section{Practice points}

- Studies are needed that meet methodological standards for diagnostic efficacy of CBCT in the diagnosis of impacted teeth

- Guidelines are needed for the use of CBCT in other clinical areas

- It will be some time before evidence is available to develop guidelines.

\section{Douglas K Benn}

Dept of General Dentistry, Creighton University School of Dentistry, Omaha, Nebraska, USA

1. Whiting P, Rutjes AW, Reitsma JB, Bossuyt PM, Kleijnen J. The development of QUADAS: a tool for the quality assessment of studies of diagnostic accuracy included in systematic reviews. BMC Med Res Methodol 2003; 3: 25-37.

2. Kapila S, Conley RS, Harrell WE. The current status of cone beam computed tomography imaging in orthodontics. Dentomaxillofac Radiol 2011; 40: 24-34.

3. Joint Position Statement of the American Association of Endodontists and the American Academy of Oral and Maxillofacial Radiology. Use of cone-beam computed tomography in endodontics. http://www.aaomr.org/resource/resmgr/Docs/AAOMRAAE_postition_paper_CB.pdf Accessed June 7, 2011.

4. Ludlow JB, Davies-Ludlow LE, White SC. Patient risk related to common dental radiographic examinations: the impact of 2007 International Commission on Radiological Protection recommendations regarding dose calculation. J Am Dent Assoc 2008; 139: 1237-1243.

5. Young SM, Lee JT, Hodges RJ, Chang TL, Elashoff DA, White SC. A comparative study of high-resolution cone beam computed tomography and charge-coupled device sensors for detecting caries. Dentomaxillofac Radiol 2009; 38: 445-451.

Evidence-Based Dentistry (2011) 12, 37. doi:10.1038/sj.ebd.6400785 